\title{
Percepción de los factores de transición a la universidad: incidencia de la realidad plural de los estudiantes
}

\author{
Montserrat Freixa-Niella ${ }^{1}$ (D), Immaculada Dorio-Alcaraz ${ }^{2}$ (D), Pilar Figura-Gazo ${ }^{2}$ (D) Mercedes Torrado- \\ Fonseca ${ }^{2}$
}

Artículo de investigación. Recibido: 02/01/2021. Aceptado: 13/05/2021. Publicación avanzada: 10/06/2021. Publicado: 01/07/2021

Resumen

INTRODUCCIÓN. Este artículo analiza la transición universitaria, en el primer semestre del primer curso, de los estudiantes de los grados de Educación Social, Educación Infantil y Relaciones Laborales de la Universidad de Barcelona. Se centra en los factores facilitadores e inhibidores de la transición y la conducta de estudio que muestran los estudiantes con diferentes trayectorias académicas previas.

MÉTODO. Se realiza un estudio descriptivo por encuesta a partir de un cuestionario (cumplimentado por 651 estudiantes) sobre las trayectorias académicas previas de los estudiantes.

RESULTADOS. Hay diferencias en las valoraciones de los estudiantes en función de su titulación y trayectoria de acceso. Los estudiantes procedentes de vías profesionalizadoras (ciclos formativos) encuentran más barreras en su proceso de transición y puntúan más alto los factores inhibidores. Se resalta el desajuste académico entre las expectativas iniciales de los estudiantes y el plan de estudios. Se enfatiza el apoyo del entorno y la relación con los compañeros como factor facilitador de la transición.

DISCUSIÓN. Los resultados aconsejan repensar las propuestas de orientación y de acción tutorial hacia modelos holísticos que permitan un contexto institucional más disponible, más flexible y con una mayor capacidad de adaptación a la pluralidad emergente de estudiantes.

\section{Palabras clave}

Universidad; Estudiante universitario; Transición secundaria superior-enseñanza superior; Estudiantes no convencionales; Factores de transición.

\section{Datos de las autoras}

${ }^{1}$ Universitat de Barcelona, España. Profesora titular en el departamento de Métodos de Investigación y Diagnóstico en Educación. Contacto para la correspondencia: mfreixa@ub.edu

${ }^{2}$ Universitat de Barcelona, España. Profesoras titulares en el departamento de Métodos de Investigación y Diagnóstico en Educación.

\section{Referencia recomendada}

Freixa-Niella, M., Dorio-Alcaraz, I., Figura-Gazo, P., y Torrado-Fonseca, M. (2021). Percepción de los factores de transición a la universidad: incidencia de la realidad plural de los estudiantes. REIRE Revista d'Innovació $i$ Recerca en Educació, 14(2), 1-17. https://doi.org/10.1344/reire2021.14.233236

(C) 2021 Las autoras. Este artículo es de acceso abierto sujeto a la licencia Reconocimiento 4.0 Internacional de Creative Commons, la cual permite utilizar, distribuir y reproducir por cualquier medio sin restricciones siempre que se cite adecuadamente la obra original. Para ver una copia de esta licencia, visite https://creativecommons.org/licenses/by/4.0/ 


\section{Títol (català)}

Percepció dels factors de transició a la universitat: incidència de la realitat plural dels estudiants

\section{Resum}

INTRODUCCIÓ. Aquest article analitza la transició universitària, en el primer semestre del primer curs, dels estudiants dels graus d'Educació Social, Educació Infantil i Relacions Laborals de la Universitat de Barcelona. Se centra en els factors facilitadors i inhibidors de la transició $\mathrm{i}$ la conducta d'estudi que mostren els estudiants amb diferents trajectòries acadèmiques prèvies.

MÈTODE. Es porta a terme un estudi descriptiu per enquesta a partir d'un qüestionari, respost per 651 estudiants, sobre les seves trajectòries acadèmiques prèvies.

RESULTATS. Hi ha diferències en les valoracions dels estudiants en funció de la titulació i la seva trajectòria d'accés a la universitat. Els estudiants que procedeixen de les vies professionalitzadores (cicles formatius) es troben amb més barreres durant el procés de transició $\mathrm{i}$ puntuen més alt els factors inhibidors. Es ressalta el desajust acadèmic entre les expectatives inicials dels estudiants i el pla d'estudis. S'emfatitza el suport de l'entorn i la relació amb els companys com a factor facilitador de la transició.

DISCUSSIÓ. Els resultats obliguen a repensar les propostes d'orientació i d'acció tutorial cap a models holístics que permetin un context institucional més disponible, més flexible i amb una major capacitat d'adaptació a la pluralitat emergent d'estudiants.

Paraules clau

Universitat; Estudiant universitari; Transició secundària superior -ensenyament superior; Estudiants no convencionals; Factors de transició.

\section{Title (English)}

Perception of factors in the transition to university: the effect of increasingly plural nature of the student body

\section{Abstract}

INTRODUCTION. This article analyses the transition to university of first-year students studying on the Social Education, Children's Education and Labour Relations degrees at the University of Barcelona. More specifically, it focuses on the facilitating and inhibiting factors in the transition to university and in the study behaviour of students from a range of academic backgrounds.

METHOD. A descriptive survey study applying a Likert-type questionnaire with 651 students, which recorded data on the students' academic pathways.

RESULTS. The students' evaluations differed according to their degree and their entry route. Students from vocational pathways (Training Cycles) encountered more barriers in their transition process and scored higher on inhibiting factors. There was a clear academic mismatch between students' initial expectations and the configuration and structure of the curriculum. Support from the environment and peer relationships were notable facilitators of the transition.

DISCUSSION. The results suggest the need to rethink the proposals for tutorial action and guidance, and to introduce holistic models that promote a more flexible institutional context with a greater capacity to adapt to the needs of an increasingly plural student body.

\section{Keywords}

University; First year students; Transition from upper secondary to higher education; Unconventional students; Transition factors. 


\section{Introducción}

En las últimas décadas en España, la formación académica de los jóvenes, una vez finalizada la enseñanza obligatoria, ha aumentado de forma considerable. La mayoría de los estudiantes deciden prolongar sus estudios accediendo a la universidad (Serrano y Soler, 2015).

En general, los estudiantes acceden a la universidad española por la vía del bachillerato ${ }^{1}$ (Troiano et al., 2017) pero el impacto del aumento de estudiantes que han cursado formación profesional se hace cada vez más evidente en distintos grados universitarios, especialmente en los grados de las Ciencias Sociales y Jurídicas (Ministerio de Educación, Cultura y Deporte [MECD], 2016). En algunos de ellos, incluso se ha invertido el perfil académico previo, pasando de uno convencional orientado al estudio (bachillerato) a otro no convencional caracterizado por seguir un itinerario formativo de índole laboral a partir de los Ciclos Formativos de Grado Superior (CFGS) (Figuera, Torrado, et al., 2015). Esta situación correspondería al patrón EMI -por sus siglas en inglés de Effectively Maintained Inequality (Lucas, 2001)-, hipótesis de la explicación de desigualdad educativa: los estudiantes con mayores ventajas escogen las opciones universitarias que en un principio les puede ofrecer más contrapartidas, por ejemplo, estudios de mayor prestigio, mejor opción de ingreso en el mercado laboral, etc. (Troiano et al., 2019).

Los estudios sobre transición universitaria han ido incorporando este perfil de estudiantes no convencionales para explicar, en conjunto, cómo afecta la heterogeneidad de estudiantes al contexto social y académico universitario (Cabrera et al., 2015; Elias y Daza, 2017). Se ha pasado de investigar a los estudiantes universitarios como grupo homogéneo a incluir la heterogeneidad del estudiantado como un factor condicionante de la persistencia y retención en el contexto universitario (Soler, 2014). Esta pluralidad de estudiantes viene mediatizada por la diversidad de itinerarios formativos recorridos en la etapa preuniversitaria. Se constata que estos perfiles no convencionales tienen más problemas de persistencia y retraso académico (Bowen et al., 2009; Cabrera et al., 2012). En España, diversas investigaciones (Figuera y Torrado, 2015; Gairín et al., 2014) apoyan estos resultados mostrando la influencia de las vías de acceso en el rendimiento y la persistencia en la universidad de estos estudiantes no convencionales. Y es que, para los estudiantes de peor rendimiento, el origen social sigue determinando su continuación en los estudios y su elección de itinerarios de mayor prestigio (Daza et al., 2019).

Esta diversidad de perfiles conlleva un afrontamiento diferente de las situaciones sociales y académicas para poder transitar con éxito a la universidad. Esta transición es importante puesto que el mayor abandono universitario se produce, precisamente, en el primer año (Figuera, 2019). Los estudiantes con perfiles no convencionales se enfrentan a factores que con mayor preponderancia pueden interferir en su progreso académico como la desmotivación, el factor económico, las expectativas, el incremento de estrés, la gestión de los diferentes roles -familiares, laborales, académicos...-, entre otros factores (Adams y Corbett, 2010; Dorio, 2017; Gilardi y Guglielmetti, 2011; Jiménez y Márquez, 2014; Tuero et al., 2018). Más concretamente, el ajuste entre las expectativas previas de los estudiantes y la realidad con la que se encuentran en la universidad ayuda a la motivación e implicación (Almeida et al., 2012; Hong et al., 2011). Asimismo, los estudiantes no convencionales presentan diferencias en las conductas de estudio y en las estrategias de aprendizaje en función de su situación de partida, más concretamente del bagaje académico previo (Langa y Rio, 2013; Rosário et al., 2014). En este sentido, cobra importancia el microcontexto académico y social, es decir, el entorno del grado que están cursando los estudiantes. Cada grado plantea

1 Las vías de acceso a la universidad española son: bachillerato, segunda carrera, estudios universitarios iniciados, Ciclos Formativos de Grado Superior y los mayores de 25,40 y 45 años. 
una estructura diferente del proceso de enseñanza-aprendizaje (Freixa et al., 2015) que puede facilitar o inhibir el proceso de transición. Así, metodologías colaborativas y más activas, donde el protagonista es el estudiante (Tinto, 2012) y el profesorado valora su experiencia vital (Rosário et al., 2014), facilitan la permanencia de los estudiantes no convencionales. El ambiente social del grado como el tipo de interacción con el profesorado y el apoyo de los compañeros del aula son factores que favorecen la implicación en el estudio, al mismo tiempo que la permanencia (Freixa et al., 2019).

Estos "nuevos estudiantes", descritos por Soler (2014) como trabajadores o desajustados, se diferencian de aquellos con un perfil convencional al mostrar actitudes periféricas, que les llevan a estar menos comprometidos académicamente puesto que priorizan otras actividades de tipo laboral o familiar; suelen ser, además, de mayor edad. Así pues, nos hallamos ante estudiantes que se relacionan con sus estudios universitarios a través de un compromiso institucional más flexible y con unas expectativas acorde a unas necesidades más personales que académicas. En cambio, los estudiantes convencionales se distinguen por ser más jóvenes (entre 18-22 años), mostrar un alto compromiso académico, estudiar a tiempo completo, asistir a clase e invertir esfuerzo en el estudio. Ahora bien, el perfil del estudiante convencional considerado hasta ahora como comprometido y vinculado a la institución y cuya actividad principal es el estudio, parece, cada vez más, estar menos presente en algunos grados universitarios.

En el caso de los grados de ciencias sociales, el acceso de estudiantes procedentes de CFGS se ha incrementado en los últimos años y, en algunas titulaciones, constituyen una mayoría. Si bien conocemos los datos globales, existen pocas investigaciones que hayan analizado la trayectoria previa ${ }^{2}$ de estos estudiantes. Y, más todavía, en qué medida esta trayectoria puede afectar sus percepciones en relación con los factores de transición a la universidad. Por este motivo, en este artículo se analizan las trayectorias académicas previas de los estudiantes antes de acceder a la universidad y su relación con las percepciones sobre los factores de influencia en la transición, incluida la conducta hacia el estudio. Para ello se han seleccionado tres titulaciones de ciencias sociales que comparten como característica una alta presencia de estudiantes de CFGS: el grado de Educación Social, maestro de Educación Infantil y Relaciones Laborales.

\section{Método}

La investigación ha permitido conocer la transición de los estudiantes de primer curso de los grados de Educación Infantil (EI) y Educación Social (ES) de la Facultad de Educación y el grado de Relaciones Laborales (RL) de la Facultad de Derecho de la Universidad de Barcelona. El acercamiento al fenómeno de la transición ha sido mediante una investigación descriptiva por encuesta y contextualizada en estas tres titulaciones del ámbito de Ciencias Sociales y Jurídicas.

Los objetivos del estudio que se expone en este artículo son:

- Identificar las trayectorias académicas previas con mayor presencia.

- Conocer los factores facilitadores e inhibidores del proceso de transición a la Universidad de estos estudiantes según la titulación y su trayectoria.

2 De hecho, la trayectoria de los estudiantes que acceden de CFGS en nuestro país puede corresponder a estudiantes procedentes de bachillerato y aquellos que proceden de un ciclo formativo de grado medio (CFGM). 
- Conocer la percepción que tienen estos estudiantes sobre su conducta de estudio según la titulación y su trayectoria.

\subsection{Instrumento de recogida de datos}

Se aplicó un cuestionario con preguntas cerradas tipo Likert de cinco puntos de valoración. El cuestionario elaborado ad hoc es una adaptación, realizada por el grupo de investigación TRALS de dos cuestionarios: Cuestionario sobre la Integración Inicial en la Universidad (CIIU) y Cuestionario de Valoración del Primer Semestre (CVPS).

El cuestionario recoge los datos personales que describen la muestra, las trayectorias académicas previas, las valoraciones de los factores sociocognitivos según el modelo teórico de R. Lent (Figuera, 2015; Lent et al., 2009); las valoraciones de los factores facilitadores e inhibidores de la transición y la conducta hacia el estudio durante el primer semestre del primer curso. En este artículo se profundiza en estos dos últimos constructos.

La escala de factores facilitadores e inhibidores de la persistencia universitaria es una escala elaborada por el equipo de investigación TRALS. Esta escala pretende evaluar las atribuciones que hacen los estudiantes de situaciones personales, contextuales y sociales en su persistencia: capacidad personal, motivación personal interacción social, trabajo, hábitos de estudios, calidad docente, etc. La escala original de 11 ítems presentaba una consistencia interna aceptable (alfa de Cronbach $=0,795$ ).

La escala de conducta hacia el estudio recoge la percepción del estudiante respecto a su capacidad para gestionar y organizar el estudio, por una parte, y por la otra, su conducta como estudiante y sus estrategias y actividades para el aprendizaje. Es una adaptación de la escala de valoración (Estrategias y actividades de estudio) del cuestionario original de Abandono de Estudios Universitarios (Bethencourt et al., 2008). La escala adaptada por Figuera (2015) de 8 ítems presenta un valor del alfa de Cronbach de 0,70 .

Para identificar las trayectorias académicas previas se elaboró una pregunta semi-abierta con las distintas posibilidades de itinerarios formativos que han seguido los estudiantes antes de acceder a la universidad, más allá de los tradicionales.

\subsection{Población y muestra}

La población está formada por el total de estudiantes matriculados $(N=651)$ del primer curso del año académico 2013-2014. Tal y como muestra la tabla 1, la población de estudio se distribuye entre las titulaciones de Educación Infantil (EI, N = 212), de Educación Social (ES, N =192) y de Relaciones Laborales ( $R L, N=247$ ). A fin de garantizar la máxima participación de estos estudiantes, se consensuó, con los responsables de las titulaciones, el momento (día y hora) más adecuado para cumplimentar el cuestionario al inicio del segundo semestre del primer curso. Asimismo, se solicitaron los permisos de consentimiento de participación oportunos tanto a la institución como a los propios estudiantes. Finalmente, participaron aquellos estudiantes que asistieron al aula en el momento de la administración del cuestionario. 
La participación, siendo voluntaria, registró un alto nivel en El $(75,47 \%)$ y en ES $(79,19 \%)$ y un porcentaje menor en RL $(59,61 \%)$. El elevado porcentaje de participación garantiza la representatividad de la población y cuantifica su error muestral en $2,5 \%$ con un nivel de confianza del $95 \%$.

Tabla 1

Distribución de la población y muestra por titulaciones

\begin{tabular}{lcccc}
\hline Titulación & Población & Muestra & Tasa de participación & Error muestral* \\
\hline Educación Infantil & 212 & 159 & 75,47 & $3,9 \%$ \\
Educación Social & 192 & 151 & 79,17 & $3,7 \%$ \\
Relaciones Laborales & 247 & 147 & 59,51 & $5,3 \%$ \\
\hline TOTAL & 651 & 457 & 70,51 & $2,5 \%$
\end{tabular}

*Nota: ( $p, q=0,5$; nivel de confianza $95 \%$ ). Fuente: Elaboración propia.

El perfil de los estudiantes en las tres titulaciones es similar. Son carreras feminizadas especialmente en El y ES y más del $60 \%$ en RL. El promedio de edad de los estudiantes está comprendido entre 21 (EI) y 22 años (ES y RL).

\subsection{Análisis de los datos}

El análisis de los datos se ha realizado con el programa informático de estadística SPSS v.20. Se ha verificado que las variables cuantitativas analizadas no cumplen los supuestos paramétricos, por lo que se ha utilizado pruebas de contraste no paramétricas como $\mathrm{H}$ de Kruskal-Wallis.

\section{Resultados}

Se aborda primero las trayectorias académicas previas de los estudiantes antes de acceder al grado. Seguidamente, se comparan los estudiantes de los diferentes grados y según su trayectoria con los factores facilitadores e inhibidores de la persistencia universitaria y con la conducta de estudio.

\subsection{Trayectorias académicas previas de los estudiantes que acceden al grado}

A partir de la información recogida sobre las trayectorias académicas previas al acceso a la universidad se ha podido constatar la existencia de itinerarios de ingreso diferentes a los denominados convencionales como el de la vía de bachillerato. En concreto, se han delimitado tres trayectorias académicas previas con más presencia.

En la primera trayectoria, la mayoritaria, los estudiantes proceden de bachillerato. En la segunda se encuentran los estudiantes que, después de aprobar el bachillerato, cursan un ciclo formativo de grado superior (CFGS) antes de entrar en la Universidad. La tercera está formada por los estudiantes que acceden a la universidad habiendo cursado primero un ciclo formativo de grado medio (CFGM) y después un ciclo formativo de grado superior (CFGS). Se incluye esta última trayectoria puesto que visibiliza una realidad que en las vías de acceso oficiales no se contempla, al ser estos estudiantes contabilizados como procedentes de la trayectoria del CFGS. Sin embargo, el bagaje académico 
previo de estos estudiantes no es el mismo, puesto que no han cursado el bachillerato. De ahí, el interés de esta última trayectoria.

La comparación de las tres titulaciones presente en la tabla 2 muestra que la trayectoria de Bachillerato es mayoritaria en el grado de RL $(48,9 \%)$ y similar en los grados de ES $(33,7 \%)$ y EI $(32,7 \%)$. La trayectoria 2 CFGS predomina en El $(50,3 \%)$, seguida en ES $(35,7 \%)$ y en un menor porcentaje en RL (23,1 \%). La trayectoria 3 CFGM + CFGS obtiene el mismo porcentaje en RL (12,9\%) y ES $(12,6 \%)$, y en menor medida, en el grado de maestro de EI (5,6\%). Finalmente, la trayectoria "otros", formada por estudiantes mayores de 25,40 y 45 años, con otros grados finalizados, con estudios universitarios iniciados, no se han analizado por tener en cada una de sus categorías una muestra muy baja.

Tabla 2

Distribución de la muestra por titulaciones y trayectorias

\begin{tabular}{llll}
\hline & $\begin{array}{l}\text { Educación Infantil } \\
\mathbf{N}=159\end{array}$ & $\begin{array}{l}\text { Educación Social } \\
\mathbf{N}=151\end{array}$ & $\begin{array}{l}\text { Relaciones Laborales } \\
\mathbf{N}=147\end{array}$ \\
\hline Trayectoria 1 BACHILLERATO & $\mathrm{n}=51(32,07 \%)$ & $\mathrm{n}=51(33,7 \%)$ & $\mathrm{n}=72(48,9 \%)$ \\
Trayectoria 2 CFGS & $\mathrm{n}=80(50,3 \%)$ & $\mathrm{n}=54(35,7 \%)$ & $\mathrm{n}=34(23,1 \%)$ \\
Trayectoria 3 CFGM + CFGS & $\mathrm{n}=9(5,6 \%)$ & $\mathrm{n}=19(12,6 \%)$ & $\mathrm{n}=19(12,9 \%)$ \\
Otras trayectorias & $\mathrm{n}=19(12,03 \%)$ & $\mathrm{n}=17(18 \%)$ & $\mathrm{n}=22(15,1 \%)$
\end{tabular}

Fuente: Elaboración propia.

\subsection{Factores facilitadores e inhibidores de la persistencia universitaria}

Los factores de esta escala se refieren a: contenido de las asignaturas, desarrollo de la docencia, organización del plan de estudios, exigencia académica del grado, hábitos de estudio, organización y dedicación al estudio, acontecimientos personales o familiares, situación económica, compañeros de clase, apoyo social y familiar, interés y preferencia por la titulación.

En términos generales, los resultados obtenidos en esta escala presentan valores moderados-altos -medias cercanas al 3,5 en una escala de 5 puntos-. Podemos afirmar pues, que los estudiantes de estas titulaciones, en su conjunto, no identifican factores específicamente inhibidores en su proceso de transición a la universidad.

Sin embargo, como muestra la tabla 3, existen diferencias significativas entre todas las valoraciones medias de los estudiantes de las tres titulaciones. Los estudiantes del grado de El y de ES aprecian como un factor inhibidor del proceso de transición que el contenido de las asignaturas no se ajuste a sus expectativas (valoración $<3$ ). Para los estudiantes de ES también es un factor inhibidor la manera en que se desarrolla la docencia (valoración < 3). Para los estudiantes de RL, el factor inhibidor es su organización y dedicación al estudio (valoración < 3).

Se identifican patrones similares en relación con los factores facilitadores en el transcurso del primer año (valoración $\geq 4$ ). Así pues, para las tres titulaciones, el apoyo social y la relación con los compañeros se perciben claramente como factores facilitadores de la transición académica durante el primer año. Así mismo, para los estudiantes de El el interés y preferencia por la titulación es considerado también un factor facilitador. 
Tabla 3

Factores facilitadores e inhibidores según el grado

\begin{tabular}{|c|c|c|c|c|c|c|c|}
\hline \multirow{2}{*}{$\begin{array}{l}\text { Factor facilitador }(\geq 4) \\
\text { Factor inhibidor }(<3)\end{array}$} & \multicolumn{2}{|c|}{$\begin{array}{c}\text { EI } \\
(n=160)\end{array}$} & \multicolumn{2}{|c|}{$\begin{array}{c}\text { ES } \\
(n=152)\end{array}$} & \multicolumn{2}{|c|}{$\begin{array}{c}R L \\
(n=147)\end{array}$} & \multirow{2}{*}{$\begin{array}{c}\text { Prueba de } \\
\text { contraste } \mathrm{H} \text { de } \\
\text { Kruskal-Wallis }\end{array}$} \\
\hline & $\bar{x}$ & d.t. & $\bar{x}$ & d.t. & $\bar{x}$ & d.t. & \\
\hline Contenido de las asignaturas & 2,81 & 0,95 & 2,67 & 1,14 & 3,30 & 1,11 & Sig. $<0,01$ \\
\hline Desarrollo de la docencia & 3,08 & 1,17 & 2,99 & 0,99 & 3,49 & 1,00 & Sig. $<0,01$ \\
\hline Organización del plan de estudios & 3,76 & 0,99 & 3,40 & 0,99 & 3.69 & 1,10 & Sig. $<0,01$ \\
\hline Exigencia académica del grado & 3,45 & 0,90 & 3,54 & 0,87 & 3,24 & 1,05 & Sig. $<0,05$ \\
\hline Hábitos de estudio & 3,89 & 0,96 & 3,53 & 1,01 & 3,13 & 1,17 & Sig. $<0,01$ \\
\hline Organización y dedicación al estudio & 3,29 & 1,05 & 3,07 & 1,14 & 2,98 & 1,13 & Sig. $<0,05$ \\
\hline $\begin{array}{l}\text { Acontecimientos } \\
\text { personales/familiares }\end{array}$ & 3,64 & 1,30 & 3,06 & 1,47 & 3,08 & 1,37 & Sig. $<0,01$ \\
\hline Situación económica & 3,74 & 1,17 & 3,38 & 1,36 & 3,24 & 1,40 & Sig. $<0,01$ \\
\hline Compañeros de clase & 4,44 & 0,88 & 4,27 & 0,99 & 4,09 & 1,04 & Sig. $<0,01$ \\
\hline Apoyo social y familiar & 4,52 & 0,78 & 4,29 & 1,01 & 4,13 & 1,14 & Sig. $<0,05$ \\
\hline Interés y preferencia por la titulación & 4,06 & 0,99 & 3,92 & 1,07 & 3,68 & 1,10 & Sig. $<0,01$ \\
\hline
\end{tabular}

Fuente: Elaboración propia.

La Tabla 4 muestra la valoración de los estudiantes según sus trayectorias. Se observa la existencia de algunas coincidencias y de algunas diferencias. Los estudiantes de las tres trayectorias coinciden en valorar como factores facilitadores (valoración $\geq 4$ ) tanto el apoyo social y familiar como la relación con los compañeros de clase. Los estudiantes de la trayectoria CFGM + GS también consideran un factor facilitador que el grado se ajuste a sus intereses y preferencias.

En cambio, sí existen diferencias en los factores inhibidores. Los estudiantes de la trayectoria CFGS valoran como factor inhibidor $(<3)$ que el contenido de las asignaturas no se ajuste a sus expectativas y para los estudiantes de la trayectoria CFGM + GS, los factores inhibidores son el desajuste en la manera de organizarse y dedicarse al estudio y la situación económica.

Por lo tanto, ¿Cuáles son los factores de transición cuando se analiza cada grado en función de las trayectorias?

Los estudiantes de El (Tabla 5), independientemente de su trayectoria académica, coindicen en determinar como factores facilitadores (valoración $\geq 4$ ) el apoyo social y familiar y la relación con los compañeros de clase. Igualmente es considerado un factor facilitador disponer de los hábitos de estudio adecuados. Para los estudiantes de la trayectoria Bachillerato no tener dificultades económicas es considerado un factor facilitador del mismo modo que los estudios se ajusten a los intereses y preferencias.

Como factores inhibidores, los estudiantes destacan, en conjunto, que el contenido de las asignaturas no se ajuste a sus expectativas. Para los estudiantes de la trayectoria CFGM + GS poder afrontar las exigencias del grado y el desarrollo de la docencia son factores inhibidores (valoración <3). 
Tabla 4

Factores facilitadores e inhibidores según los itinerarios de las trayectorias académicas previas

\begin{tabular}{|c|c|c|c|c|c|c|c|}
\hline \multirow{2}{*}{$\begin{array}{l}\text { Factor facilitador }(\geq 4) \\
\text { Factor inhibidor }(<3)\end{array}$} & \multicolumn{2}{|c|}{$\begin{array}{l}\text { Bachillerato } \\
(n=174)\end{array}$} & \multicolumn{2}{|c|}{$\begin{array}{c}\text { CFGS } \\
(n=165)\end{array}$} & \multicolumn{2}{|c|}{$\begin{array}{c}\text { CFGM + GS } \\
(n=46)\end{array}$} & \multirow{2}{*}{$\begin{array}{c}\text { Prueba de } \\
\text { contraste } \\
\text { H de Kruskal- } \\
\text { Wallis }\end{array}$} \\
\hline & $\bar{x}$ & d.t. & $\bar{x}$ & d.t. & $\bar{x}$ & d.t. & \\
\hline Contenido de las asignaturas & 3,04 & 1,06 & 2,67 & 1,08 & 3,04 & 1,15 & Sig. $<0,01$ \\
\hline Desarrollo de la docencia & 3,36 & 0,98 & 3,01 & 1,13 & 3,42 & 1,09 & Sig. $<0,01$ \\
\hline Organización del plan de estudios & 3,81 & 1,05 & 3,54 & 1,02 & 3,51 & 0,96 & Sig. $<0,05$ \\
\hline Exigencia académica del grado & 3,57 & 0,91 & 3,37 & 0,01 & 3,20 & 0,93 & Sig. $<0,05$ \\
\hline Hábitos de estudio & 3,59 & 1,18 & 3,58 & 0,94 & 3,37 & 1,21 & No sig. \\
\hline Organización y dedicación al estudio & 3,17 & 1,12 & 3,21 & 1,06 & 2,98 & 1,21 & No sig. \\
\hline Acontecimientos personales /familiares & 3,28 & 1,41 & 3,29 & 1,40 & 3,35 & 1,47 & No sig. \\
\hline Situación económica & 3,79 & 1,20 & 3,41 & 1,35 & 2,98 & 1,35 & Sig. $<0,01$ \\
\hline Compañeros de clase & 4,28 & 1,02 & 4,34 & 0,89 & 4,13 & 1,08 & No sig. \\
\hline Apoyo social y familiar & 4,45 & 0,86 & 4,28 & 1,05 & 4,29 & 1,18 & No sig. \\
\hline Interés y preferencia por la titulación & 3,87 & 1,10 & 3,84 & 1,08 & 4,02 & 1,00 & No sig. \\
\hline
\end{tabular}

Fuente: Elaboración propia.

Tabla 5

Factores facilitadores e inhibidores en el grado de maestro de Educación Infantil según la trayectoria académica

\begin{tabular}{|c|c|c|c|c|c|c|c|}
\hline \multirow{2}{*}{$\begin{array}{l}\text { Factor facilitador }(\geq 4) \\
\text { Factor inhibidor }(<3)\end{array}$} & \multicolumn{2}{|c|}{$\begin{array}{l}\text { Bachillerato } \\
\quad(n=51)\end{array}$} & \multicolumn{2}{|c|}{$\begin{array}{c}\text { CFGS } \\
(n=80)\end{array}$} & \multicolumn{2}{|c|}{$\begin{array}{c}\text { CFGM + GS } \\
(n=9)\end{array}$} & \multirow{2}{*}{$\begin{array}{c}\text { Prueba de } \\
\text { contraste } \\
\text { H de Kruskal } \\
\text { Wallis } \\
\end{array}$} \\
\hline & $\bar{x}$ & d.t. & $\bar{x}$ & d.t. & $\bar{x}$ & d.t. & \\
\hline Contenido de las asignaturas & 2,94 & 1,08 & 2,72 & 0,90 & 2,78 & 0,66 & No sig. \\
\hline Desarrollo de la docencia & 3,39 & 1,11 & 3,05 & 1,14 & 2,67 & 1,22 & No sig. \\
\hline Organización del plan de estudios & 3,80 & 1,07 & 3,80 & 0,89 & 3,67 & 1,11 & No sig. \\
\hline Exigencia académica del grado & 3,69 & 0,83 & 3,31 & 0,86 & 2,89 & 1,05 & Sig. $<0,01$ \\
\hline Hábitos de estudio & 4,02 & 1,01 & 3,80 & 0,92 & 4,11 & 1,05 & No sig. \\
\hline Organización y dedicación al estudio & 3,47 & 1,00 & 3,28 & 1,01 & 2,89 & 1,45 & No sig. \\
\hline Acontecimientos personales/familiares & 3,61 & 1,25 & 3,59 & 1,36 & 4,00 & 1,5 & No sig. \\
\hline Situación económica & 4,25 & 0,84 & 3,68 & 1,22 & 3,33 & 1,32 & Sig. $<0,05$ \\
\hline Compañeros de clase & 4,39 & 1,00 & 4,49 & 0,76 & 4,67 & 0,50 & No sig. \\
\hline Apoyo social y familiar & 4,61 & 0,69 & 4,49 & 0,84 & 4,56 & 1,01 & No sig. \\
\hline Interés y preferencia por la titulación & 4,24 & 0,92 & 3,91 & 1,04 & 3,78 & 1,09 & No sig. \\
\hline
\end{tabular}

Fuente: Elaboración propia.

En el grado de ES (Tabla 6), todos los estudiantes perciben como factores facilitadores el apoyo social y familiar y la relación con los compañeros. Para los estudiantes de la trayectoria CFGM + GS también lo es realizar unos estudios ajustados a los intereses y preferencias. 
Los estudiantes de la trayectoria Bachillerato consideran factores inhibidores el contenido de las asignaturas no ajustado a sus expectativas, las dificultades para organizarse y dedicarse al estudio y los acontecimientos personales/familiares que dificultan el proceso de transición académica. Los estudiantes de la trayectoria CFGS perciben como factores inhibidores el contenido de las asignaturas no ajustado a sus expectativas y el desarrollo de la docencia.

\section{Tabla 6}

Factores facilitadores e inhibidores en el grado de Educación Social según la trayectoria académica

\begin{tabular}{|c|c|c|c|c|c|c|c|}
\hline \multirow{2}{*}{$\begin{array}{l}\text { Factor facilitador }(\geq 4) \\
\text { Factor inhibidor }(<3)\end{array}$} & \multicolumn{2}{|c|}{$\begin{array}{l}\text { Bachillerato } \\
\quad(n=51)\end{array}$} & \multicolumn{2}{|c|}{$\begin{array}{c}\text { CFGS } \\
(n=51)\end{array}$} & \multicolumn{2}{|c|}{$\begin{array}{l}\text { CFGM + GS } \\
(n=18)\end{array}$} & \multirow{2}{*}{$\begin{array}{c}\text { Prueba de } \\
\text { contraste } \\
\text { H de Kruskal- } \\
\text { Wallis }\end{array}$} \\
\hline & $\bar{x}$ & d.t. & $\bar{x}$ & d.t. & $\bar{x}$ & d.t. & \\
\hline Contenido de las asignaturas & 2,76 & 1,03 & 2,27 & 1,18 & 3,00 & 1,08 & Sig. $<0,05$ \\
\hline Desarrollo de la docencia & 3,10 & 0,87 & 2,61 & 1,06 & 3,72 & 0,89 & Sig. $<0,01$ \\
\hline Organización del plan de estudios & 3,61 & 1,02 & 3,14 & 1,02 & 3,50 & 0,85 & Sig. $<0,05$ \\
\hline Exigencia académica del grado & 3,67 & 0,88 & 3,63 & 0,79 & 3,39 & 0,85 & No sig. \\
\hline Hábitos de estudio & 3,59 & 1,08 & 3,63 & 0,89 & 3,56 & 1,04 & No sig. \\
\hline $\begin{array}{l}\text { Organización y dedicación al } \\
\text { estudio }\end{array}$ & 2,98 & 1,14 & 3,24 & 1,11 & 3,11 & 1,27 & No sig. \\
\hline $\begin{array}{l}\text { Acontecimientos } \\
\text { personales/familiares }\end{array}$ & 2,88 & 1,47 & 3,25 & 1,48 & 3,56 & 1,33 & No sig. \\
\hline Situación económica & 3,67 & 1,22 & 3,28 & 1,41 & 3,06 & 1,34 & No sig. \\
\hline Compañeros de clase & 4,24 & 1,06 & 4,25 & 0,99 & 4,33 & 1,08 & No sig. \\
\hline Apoyo social y familiar & 4,45 & 0,75 & 4,22 & 1,16 & 4,50 & 1,04 & No sig. \\
\hline $\begin{array}{l}\text { Interés y preferencia por la } \\
\text { titulación }\end{array}$ & 3,84 & 1,10 & 3,82 & 1,14 & 4,39 & 0,69 & No sig. \\
\hline
\end{tabular}

Fuente: Elaboración propia.

En el grado de RL (Tabla 7), las valoraciones de los estudiantes no se distinguen por su itinerario excepto en un factor, la situación económica. Los estudiantes valoran como factores facilitadores el apoyo social y familiar y la relación con los compañeros de clase, aunque sea más evidente en los estudiantes de las trayectorias Bachillerato y CFGS.

Respecto a los factores inhibidores, cabe destacar las dificultades de ajuste académico en la trayectoria CFGS y en la CFGM + GS los hábitos de estudio y la organización y dedicación al estudio. Finalmente, hay que destacar que tanto los acontecimientos personales/familiares como la situación económica, este último factor de manera significativa, son percibidos como inhibidores del proceso de transición especialmente por estos mismos estudiantes. 


\section{Tabla 7}

Factores facilitadores e inhibidores en el grado de Relaciones Laborales según la trayectoria académica

\begin{tabular}{|c|c|c|c|c|c|c|c|}
\hline \multirow{2}{*}{$\begin{array}{l}\text { Factor facilitador }(\geq 4) \\
\text { Factor inhibidor }(<3)\end{array}$} & \multicolumn{2}{|c|}{$\begin{array}{l}\text { Bachillerato } \\
(n=72)\end{array}$} & \multicolumn{2}{|c|}{$\begin{array}{c}\text { CFGS } \\
(n=34)\end{array}$} & \multicolumn{2}{|c|}{$\begin{array}{c}\text { CFGM + GS } \\
(n=19)\end{array}$} & \multirow{2}{*}{$\begin{array}{c}\text { Prueba de } \\
\text { contraste } \\
\text { H de Kruskal- } \\
\text { Wallis }\end{array}$} \\
\hline & $\bar{x}$ & d.t. & $\bar{x}$ & d.t. & $\bar{x}$ & d.t. & \\
\hline Contenido de las asignaturas & 3,31 & 1,02 & 3,15 & 1,12 & 3,21 & 1,39 & No sig. \\
\hline Desarrollo de la docencia & 3,52 & 0,91 & 3,55 & 1,00 & 3,50 & 1,09 & No sig. \\
\hline Organización del plan de estudios & 3,96 & 1,04 & 3,55 & 1,14 & 3,44 & 1,04 & No sig. \\
\hline Exigencia académica del grado & 3,42 & 0,98 & 3,09 & 1,1 & 3,16 & 0,95 & No sig. \\
\hline Hábitos de estudio & 3,29 & 1,19 & 2,97 & 0,81 & 2,84 & 1,25 & No sig. \\
\hline $\begin{array}{l}\text { Organización y dedicación al } \\
\text { estudio }\end{array}$ & 3,10 & 1,16 & 3,03 & 1,10 & 2,88 & 1,05 & No sig. \\
\hline $\begin{array}{l}\text { Acontecimientos } \\
\text { personales/familiares }\end{array}$ & 3,34 & 1,43 & 2,64 & 1,14 & 2,84 & 1,50 & No sig. \\
\hline Situación económica & 3,55 & 1,32 & 2,94 & 1,48 & 2,74 & 1,40 & Sig. $<0,05$ \\
\hline Compañeros de clase & 4,24 & 1,00 & 4,09 & 0,98 & 3,68 & 1,15 & No sig. \\
\hline Apoyo social y familiar & 4,34 & 1,03 & 3,85 & 1,22 & 3,94 & 1,34 & No sig. \\
\hline $\begin{array}{l}\text { Interés y preferencia por la } \\
\text { titulación }\end{array}$ & 3,62 & 1,15 & 3,67 & 1,08 & 3,79 & 1,13 & No sig. \\
\hline
\end{tabular}

Fuente: Elaboración propia.

\subsection{Conducta de estudio}

La conducta de estudio se define por aquellos comportamientos que el estudiante ha adquirido durante su trayectoria académica y que le permiten afrontar con cierto éxito las demandas académicas exigidas en la titulación universitaria. Por un lado, encontramos los comportamientos que comprenden su capacidad para gestionar y organizarse en el estudio: saber gestionar el trabajo y el estudio; llevar al día las tareas y el estudio académico; juntarse con los compañeros para estudiar y llevar a cabo las tareas académicas y; revisar la gestión del trabajo para optimizarla. Por otro, se sitúan los comportamientos que reflejan el compromiso e implicación de los estudiantes por los estudios que están realizando: tener en cuenta las orientaciones del profesorado para preparar la asignatura; plantear dudas al profesorado cuando se considera pertinente; aprovechar las indicaciones del tutor/a y; asistir habitualmente a clase.

Los datos de la tabla 8 muestran que entre los estudiantes de las tres titulaciones existen diferencias significativas tanto en los ítems relacionados con la gestión y organización del estudio, excepto el ítem G4_revisar la gestión del trabajo para optimizarla, como los ítems relacionados con el compromiso e implicación hacia el estudio, excepto el ítem C3_Aprovechar las indicaciones del tutor/a. Los estudiantes de El valoran como más positiva su conducta de estudio aspecto que repercute más favorablemente en su integración académica. 


\section{Tabla 8}

La conducta de estudio según la titulación

\begin{tabular}{|c|c|c|c|c|c|c|c|}
\hline \multirow{2}{*}{$\begin{array}{l}\text { Conducta de estudio } \\
\text { (poco adecuada } 1 \text { a muy adecuada 5) }\end{array}$} & \multicolumn{2}{|c|}{$\begin{array}{c}\text { El } \\
(n=160)\end{array}$} & \multicolumn{2}{|c|}{$\underset{(n=152)}{E S}$} & \multicolumn{2}{|c|}{$\begin{array}{c}R L \\
(n=147)\end{array}$} & \multirow{2}{*}{$\begin{array}{c}\text { Prueba de } \\
\text { contraste } \mathrm{H} \text { de } \\
\text { Kruskal-Wallis }\end{array}$} \\
\hline & $\bar{x}$ & d.t. & $\bar{x}$ & d.t. & $\bar{x}$ & d.t. & \\
\hline G1_Saber gestionar el trabajo y estudio & 3,89 & 0,70 & 3,49 & 0,86 & 3,51 & 0,88 & Sig. $<0,01$ \\
\hline G2_Llevar al día las tareas y estudio académico & 3,90 & 0,79 & 3,34 & 0,94 & 3,52 & 0,91 & Sig. $<0,01$ \\
\hline $\begin{array}{l}\text { G3_Juntarse con los compañeros para estudiar y } \\
\text { llevar a cabo las tareas }\end{array}$ & 4,11 & 0,82 & 3,57 & 1,07 & 3,28 & 1,15 & Sig. $<0,01$ \\
\hline G4_Revisar la gestión del trabajo para optimizarla & 3,88 & 0,83 & 3,89 & 0,85 & 3,64 & 1,02 & No sig. \\
\hline $\begin{array}{l}\mathrm{C} 1 \_ \text {Tener en cuenta las orientaciones del } \\
\text { profesorado para preparar la asignatura }\end{array}$ & 4,11 & 0,64 & 3,87 & 0,79 & 3,84 & 0,85 & Sig. $<0,01$ \\
\hline C2_Plantear dudas al profesorado & 3,82 & 0,83 & 3,54 & 0,88 & 3,36 & 1,09 & Sig. $<0,01$ \\
\hline C3_Aprovechar las indicaciones del tutor/a & 3,54 & 0,94 & 3,33 & 0,98 & 3,54 & 0,97 & No sig. \\
\hline C4_Asistir habitualmente a clase & 4,39 & 0,65 & 3,86 & 1,03 & 3,94 & 1,06 & Sig. $<0,01$ \\
\hline
\end{tabular}

Fuente: Elaboración propia.

Y en relación con la trayectoria académica previa, la tabla 9 muestra las diferencias significativas en algunos de los ítems. Los estudiantes de la trayectoria CFGM + GS valoran en menor medida su capacidad para G1-saber gestionar el trabajo y estudio. Igualmente tienden, significativamente, a G3-no juntarse con sus compañeros para estudiar y realizar las tareas académicas. Respecto a los ítems relacionados con el compromiso e implicación al estudio, los estudiantes de las trayectorias CFGS y CFGM+GS tienen en cuenta de manera significativa C1-las orientaciones del profesorado. De la misma manera, estos estudiantes tienden a C2-plantear más dudas a sus profesores si lo consideran pertinente.

Tabla 9

La conducta de estudio según el itinerario académico previo

\begin{tabular}{|c|c|c|c|c|c|c|c|}
\hline \multirow{2}{*}{$\begin{array}{l}\text { Conducta de estudio } \\
\text { (Poco adecuada } 1 \text { a muy adecuada 5) }\end{array}$} & \multicolumn{2}{|c|}{$\begin{array}{l}\text { Bachillerato } \\
(n=174)\end{array}$} & \multicolumn{2}{|c|}{$\begin{array}{l}\text { CFGS } \\
(n=165)\end{array}$} & \multicolumn{2}{|c|}{$\begin{array}{c}\text { CFGM + GS } \\
(n=46)\end{array}$} & \multirow{2}{*}{$\begin{array}{c}\text { Prueba de } \\
\text { contraste } \mathrm{H} \text { de } \\
\text { Kruskal-Wallis }\end{array}$} \\
\hline & $\bar{x}$ & d.t. & $\bar{x}$ & d.t. & $\bar{x}$ & d.t. & \\
\hline G1_Saber gestionar el trabajo y estudio & 3,63 & 0,89 & 3,76 & 0,69 & 3,40 & 0,93 & Sig. $<0,05$ \\
\hline G2_Llevar al día las tareas y estudio académico & 3,62 & 0,89 & 3,68 & 0,82 & 3,47 & 1,01 & No sig. \\
\hline $\begin{array}{l}\text { G3_Juntarse con los compañeros para estudiar } \\
\text { y llevar a cabo las tareas }\end{array}$ & 3,62 & 1,07 & 3,90 & 0,95 & 3,44 & 1,13 & Sig. $<0,01$ \\
\hline $\begin{array}{l}\text { G4_Revisar la gestión del trabajo para } \\
\text { optimizarla }\end{array}$ & 3,77 & 0,96 & 3,85 & 0,81 & 3,64 & 1,15 & No sig. \\
\hline $\begin{array}{l}\text { C1_Tener en cuenta las orientaciones del } \\
\text { profesorado para preparar la asignatura }\end{array}$ & 3,85 & 0,75 & 4,01 & 0,74 & 4,00 & 0,97 & Sig. $<0,05$ \\
\hline C2_Plantear dudas al profesorado & 3,40 & 0,95 & 3,69 & 0,92 & 3,58 & 0,94 & Sig. $<0,05$ \\
\hline C3_Aprovechar las indicaciones del tutor/a & 3,46 & 0,95 & 3,50 & 0,98 & 3,44 & 0,91 & No sig. \\
\hline C4_Asistir habitualmente a clase & 4,07 & 0,97 & 4,18 & 0,83 & 3,71 & 1,16 & No sig. \\
\hline
\end{tabular}

Fuente: Elaboración propia. 


\section{Conclusiones y discusión}

Los analistas nacionales e internacionales sobre la educación superior coinciden en destacar la democratización del sistema en las últimas décadas, al amparo de las políticas de equidad implementadas. Una de las consecuencias ha sido el incremento del número de estudiantes no convencionales. Este estudio apoya los resultados de la literatura especializada (Lorenzo et al., 2014; Soler, 2014) y los informes de la Organización para la Cooperación y el Desarrollo Económicos (OCDE, 2017) sobre la heterogeneidad del estudiantado en las aulas universitarias, especialmente en los grados de ciencias sociales y jurídicas. Los hallazgos obtenidos confirman el incremento significativo de estudiantes procedentes de la vía profesional, revelando una nueva trayectoria: el estudiante que inicia la enseñanza postobligatoria con un ciclo formativo de grado medio y prosigue su formación con un ciclo formativo de grado superior sin haber pasado por el bachillerato. Esta situación se constata, en mayor medida, en los grados de ES y RL. Los estudiantes de la trayectoria bachillerato se van diluyendo frente al aumento de la trayectoria CFGS que ya supone el $50 \%$ en El y la nueva trayectoria CFGM + GS más presente en ES y RL.

Esta situación muestra que la desigualdad educativa continúa presente a través de las trayectorias académicas previas que conviven en la misma aula. El acceso más equitativo a la Universidad se ha producido solamente en algunos grados como los estudiados en este artículo, tal como sucede en los estudios de las ciencias sociales y jurídicas $(M E C, 2016)$. Son estudios con un alto grado de profesionalización y de inserción laboral al acabar, pero no son considerados de prestigio y tienen poca promoción en la carrera profesional.

La trayectoria académica previa explica el incremento en la media de edad de los estudiantes: 21-22 años frente a 18-19 años en contextos universitarios con estudiantes de la vía de bachillerato. Variables como el contexto socioeconómico familiar y la dedicación laboral también se vinculan con la trayectoria previa y el tipo de carreras tal como indican Sánchez-Gelabert y Elías (2017) y Troiano et al. (2017).

Los resultados sobre los factores facilitadores e inhibidores del proceso de transición durante el primer curso coinciden con otras investigaciones (Dorio, 2017; Figuera y Torrado, 2015). Es importante destacar que, independientemente del grado o de la trayectoria académica previa, la percepción de apoyo social y familiar y la relación con los compañeros de clase son consideradas, unánimemente, como factores facilitadores. Es una percepción común que va más allá del contexto, de la edad y del género. Desde un punto de vista institucional, se convierte en uno de los puntos de partida para potenciar la integración del estudiante y su persistencia en el sistema. De ahí que el grupo clase pueda ser el contexto diana para las acciones de los planes de acción tutorial (Tinto, 2012).

La investigación también muestra cómo la naturaleza del contexto académico y disciplinar condiciona, significativamente, el proceso de transición de los estudiantes, aspecto destacado en la investigación de campo (Figuera, 2019; Tinto, 2017). Así pues, los grados estudiados, presentan algunas diferencias. Por un lado, los estudiantes de maestro de El y de ES son más sensibles al desajuste entre sus expectativas en cuanto al contenido y dinámica de clases y lo que en realidad sucede en el aula, es decir, factores de tipo institucional. En cambio, los estudiantes de RL son más sensibles a los desajustes de tipo académico, verbigracia, a la capacidad para gestionar su dedicación al estudio. Por otro, las trayectorias académicas previas establecen unas diferencias estadísticamente significativas respecto a los factores que dificultan el proceso de transición durante el primer año universitario. Así, para los estudiantes de la trayectoria CFGM + GS, la situación económica se percibe como un factor que dificulta la continuidad de los estudios universitarios. Generalmente, estos estudiantes provienen de un contexto socioeconómico que les fuerza 
a financiar sus estudios trabajando (Figuera, Torrado, et al., 2015; García-Gómez y Blanco, 2015), teniendo más dificultades para su propia organización y dedicación al estudio, aspecto relacionado con su bagaje académico previo para afrontar las exigencias académicas. Finalmente, los estudiantes de maestro de EI ponen de manifiesto la relevancia de acceder a un grado que se ajuste a sus preferencias e intereses. En conclusión, se reafirma la importancia de la orientación antes de acceder a la universidad para, por una parte, profundizar en todos aquellos factores que convergen en la elección de la carrera (Figuera, Rodríguez y Llanes, 2015) y, por otra, para prestar más atención a los estudiantes de las trayectorias CFGS y CFGM + GS.

Esta discusión nos conduce finalmente al último aspecto tratado relativo a la conducta de estudio. Se vuelve a constatar la determinación del contexto académico-disciplinar relacionado con la conducta de estudio percibida por los estudiantes. Los resultados muestran diferencias significativas entre los estudiantes que consideran su conducta de estudio más ajustada para afrontar las exigencias académicas de su grado como sucede con los estudiantes de El más habituados al estudio académico en relación con los estudiantes de ES y los de RL. Parece evidente que cuando el estudiante conecta significativamente con el contenido, la metodología docente, la relación con el profesorado, entre otros factores intrínsecos como la motivación, la percepción de eficacia académica es mayor (Freixa et al., 2015).

El análisis comparativo de los resultados con relación a las trayectorias académicas previas confirma diferencias en cuanto a las percepciones y las conductas de estudio. Las diferencias más significativas se encuentran entre los estudiantes de la trayectoria CFGM + CFGS y los de la trayectoria de Bachillerato. Los resultados evidencian la existencia de la desigualdad académica previa al acceder a la universidad de los estudiantes que inician la enseñanza postobligatoria con un CFGM.

Aquellos estudiantes que provienen de las trayectorias de bachillerato y CFGS presentan aspectos coincidentes. Tal como han señalado algunas investigaciones, el contexto universitario estaría planificado y organizado, desde una lógica académica tradicional, y por lo tanto los estudiantes convencionales son los que mejor se ajustan a las exigencias académicas del grado y, además, muestran una mayor autonomía (Figuera et al., 2015). En cambio, los estudiantes cuyas trayectorias son no convencionales, como los estudiantes que provienen del $C F G M+G S$, presentan mayores lagunas académicas y requieren y demandan más apoyo académico.

Este dato nos lleva a reflexionar hasta qué punto el sistema universitario está verdaderamente comprometido con los conceptos de dimensión social y de equidad presentes en todas las agendas de las universidades y que han facilitado el acceso de estudiantes con perfiles cada vez más diversos en cuanto a trayectorias académicas previas. Este compromiso significa poder acoger a los estudiantes no convencionales que suelen tener más consolidada su elección, pues el inicio de la formación universitaria viene precedido ya por estudios similares al ámbito disciplinario desde una perspectiva más profesionalizadora, como sucede en el caso de los grados estudiados. Las dificultades expresadas por estos estudiantes permiten aventurar que la universidad aún no es sensible a la diversidad del alumnado y parece no valorar suficientemente un perfil de estudiantes que, a pesar de su motivación, no se ajustarían al estatus quo. La Universidad debe dar respuesta a estos estudiantes en el acceso, pero a partir de aquí debe poner mecanismos para que la transición del primer año sea exitosa y lleguen a su graduación, asumiendo con ello el reto de la verdadera equidad participativa. 


\section{$<$ Organismo colaborador >}

Este trabajo forma parte del proyecto "Model d'actuació acadèmic-docent per l'adaptació a la Universitat dels estudiants amb un perfil no tradicional. El cas d'educació social i relacions laborals de la UB" financiado por el Programa de Recerca en Docència Universitària (REDICE) de la Universitat de Barcelona. Redice141485.

\section{Referencias}

Adams, J., y Corbett, A. (2010). Experiences of traditional and nontraditional college students. Perspectives, 2(1), 1-29. https://scholars.unh.edu/perspectives/vol2/iss1/2

Almeida, L., Guisande, A., y Paisana, J. (2012). Extra-curriculum involvement, academic adjustment and achievement in higher education: A study of Portuguese students. Anales de Psicología, 28(3), 860865. https://doi.org/10.6018/analesps.28.3.156111

Bethencourt, J., Cabrera., Hernández, J., Álvarez, P., y González, M. (2008). Variables psicológicas y educativas en el abandono universitario. Revista Electrónica de Investigación Psicoeducativa, 6(16), 603-622. https://doi.org/10.25115/ejrep.v6i16.1298

Bowen, W., Chingos, M., y McPherson, M. (2009). Crossing the finish line: Completing College at America's Public Universities. Princeton University Press.

Cabrera, A., Burkum, K., y La Nasa, S. (2012). Pathways to a four-year degree: Determinants of degree completion among socio-economically disadvantaged students. En A. Seidman (ed.), College Student Retention: A Formula for Student Success. Praeger Publishers.

Daza, L., Troiano, H., y Elias, M. (2019). La transición a la universidad desde el bachillerato y desde el CFGS. La importancia de los factores socioeconómicos. Papers: Revista de sociología, 104(3), 425-445. https://doi.org/10.5565/rev/papers.2546

Dorio, I. (2017). La transición a la Universidad. El grado de maestro de Educación Infantil (tesis doctoral, Universitat de Barcelona). http://hdl.handle.net/2445/109484

Elias, M., y Daza, L. (2017). ¿Cómo deciden les jóvenes la transición a la educación postobligatoria? Diferencias entre centros públicos y privados-concertados. RASE Revista de la Asociación de Sociología de la Educación, 10(1), 5-22. https://doi.org/10.7203/RASE.10.1.9135

Figuera, P. (ed., 2015). Persistir con éxito en la universidad: de la investigación a la acción. Laertes.

Figuera, P. (ed., 2019). Trayectorias, transiciones y resultados de los estudiantes en la universidad. Laertes.

Figuera, P., Rodríguez, L., y Llanes, J. (2015). Transición y orientación: interrelaciones, estrategias y recomendaciones desde la investigación. REIRE Revista d'Innovació i Recerca en Educació, 8(2), 117. https://doi.org/10.1344/reire2015.8.2821

Figuera, P., y Torrado, M. (2015). The Transition to University of At-Risk Groups in Spain: The Case of Students from Vocational Education and Training. Revista de Cercetare Si Interventie Sociala 49, 23-40. https://www.rcis.ro/images/documente/rcis49_02.pdf 
Figuera, P., Torrado, M., Freixa, M., y Dorio, I. (2015). Trayectorias de persistencia y abandono de estudiantes no convencionales: implicaciones para la orientación. Revista Electrónica Interuniversitaria de Formación del Profesorado, 18(2), 107-123. https://doi.org/10.6018/reifop.18.2.220101

Freixa, M., Aparicio-Chueca, P., y Triadó, X. (2015). El rol del profesorado como elemento clave en las instituciones y el contexto de la educación superior. En P. Figuera (ed.), Persistir con éxito en la universidad: De la investigación a la acción. Laertes.

Freixa, M., Dorio, I., y Corti, F. (2019). First Year Students' Perceptions in Transition to University: The Students' Perceptions. Multidisciplinary Journal of School Education, 2(16), 25-56. https://doi.org/10.14632/mjse.2019.16.25

Gairín, J., Triado, X., Feixas, M., Figuera, P., Aparicio-Chueca, P., y Torrado, M. (2014). Student dropout rates in Catalan universities: profile and motives for disengagement. Quality in Higher Education, 20(2), 165-182. https://doi.org/10.1080/13538322.2014.925230

García-Gómez, S., y Blanco, N. (2015). Los ciclos formativos de grado medio: una opción para salir del laberinto personal y académico. Tendencias Pedagógicas, 25, 301-320. https://hdl.handle.net/10486/663436

Gilardi, S., y Guglielmetti, C. (2011). University life of non-traditional students: Engagement styles and impact on attrition. Journal of Higher Education, 82(1), 33-53. https://doi.org/10.1080/00221546.2011.11779084

Hong, B., Shull, P., y Haefner, L. (2011). Impact of perceptions of faculty on student outcomes of selfefficacy, locus of control, persistence, and commitment. Journal of College Students Retention: Research, Theory and Practice, 13, 289-309. https://doi.org/10.2190/CS.13.3.b

Jiménez, M-L., y Márquez, E. (2014). Ir a la universidad después de los 30: dificultades y factores facilitadores. Aula Abierta, 42(1), 1-8. https://doi.org/10.1016/S0210-2773(14)70001-0

Langa, D., y Río, M-A. (2013). Los estudiantes de clases populares en la universidad y frente a la universidad de la crisis: persistencia y nuevas condiciones para la multiplicación de la desigualdad de oportunidades educativas. Tempora: Revista de Sociología de la Educación, 16, 71-96. http://riull.ull.es/xmlui/handle/915/4744

Lent, R-W., Taveira, M-C., Singley, D., Sheu, H., y Hennessy, K. (2009). Social cognitive predictors of academic adjustment and live satisfaction in Portuguese college students: A longitudinal analysis. Journal of Vocational Behaivor, 74, 190-198. https://doi.org/10.1016/j.jvb.2008.12.006

Lucas, S. (2001). Effectively Maintained Inequality: Education Transitions, Track Mobility, and Social Background Effects. American Journal of Sociology, 106(6), 1642-1690. https://doi.org/10.1086/321300

Lorenzo, M., Argos, J., Hernández, J., y Vera, J. (2014). El acceso y la entrada del estudiante a la universidad: Situación y propuestas de mejora facilitadoras del tránsito. Educación XX1, 17(1), 15-38. https://doi.org/10.5944/educxx1.17.1.9951 
Ministerio de Educación, Cultura y Deporte. (2016). Datos básicos del sistema universitario español. Curso 15-16.http://www.educacionyfp.gob.es/dam/jcr:6a538b72-1866-4100-b1b7b3fd26bb8191/datos-y-cifras-sue-2015-16-web-.pdf

Organización para la Cooperación y el Desarrollo Económico. (2017). Panorama de la Educación. Informe Español. https://www.mecd.gob.es/dctm/inee/eag/2017/panorama-de-la-educacion-2017-def12-09-2017red.pdf?documentld=0901e72b8263e12d

Rosário, P., Pereira, A., Núñez, J-C., Cunha, J., Fuentes, S., Polydoro, S., Gaeta, M., y Fernández, E. (2014). An explanatory model of the intention to continue studying among nontraditional university students. Psycothema, 26(1), 84-90. https://doi.org/10.7334/psicothema2013.176

Sánchez-Gelabert, A., y Elías, M. (2017). Los estudiantes universitarios no tradicionales y el abandono de los estudios. Estudios sobre educación, 32, 27-48. https://doi.org/10.15581/004.32.27-48

Serrano, L., y Soler, A. (2015). La formación y el empleo de los jóvenes españoles. Trayectoria reciente y escenarios futuros. Fundación BBVA.

Soler, I. (2014). Una tipología de la población estudiantil universitaria. RASE Revista de Sociología de la Educación, 7(1), 104-122. https://ojs.uv.es/index.php/RASE/article/view/10191

Tinto, V. (2012). Completing college: Rethinking institutional action. University of Chicago Press.

Tinto, V. (2017). Through the eyes of students. Journal of College Student Retention: Research, Theory and Practice, 19(3), 254-269. https://doi.org/10.1177/1521025115621917

Troiano, H., Sánchez-Gelabert, A., Torrents, D., Elías, M., y Daza, L. (2019). Estudios sobre trayectorias y transiciones de los estudiantes universitarios. Una perspectiva sociológica. En Pilar Figuera Gazo (ed.), Trayectorias, transiciones y resultados de los estudiantes en la universidad (pp. 31-70). Laertes.

Troiano, H., Torrents, D., Sánchez-Gelabert, A., y Daza, L. (2017). Evolución del acceso a la universidad y de la elección de titulación universitaria entre la población joven en Catalunya. Cuadernos de Relaciones Laborales, 35(2), 281-303. https://doi.org/10.5209/CRLA.56775

Tuero, E., Cervero, A., Esteban, M., y Bernardo, A. (2018). ¿Por qué abandonan los alumnos universitarios? Variables de influencia en el planteamiento y consolidación del abandono. Educación XX1, 21(2), 131-154. https://doi.org/10.5944/educXX1.20066 